\title{
Stochastic entropies and fluctuation theorems for a discrete one-dimensional Kardar-Parisi-Zhang system
}

\author{
Miguel A. Rodríguez $\oplus^{1}$ and Horacio S. Wio ${ }^{2}$ \\ ${ }^{1}$ Instituto de Física de Cantabria (IFCA), CSIC-UNICAN, E-39005 Santander, Spain \\ ${ }^{2}$ Instituto de Física Interdisciplinar y Sistemas Complejos (IFISC), CSIC-UIB, E-07122 Palma de Mallorca, Spain
}

(Received 29 March 2019; revised manuscript received 31 May 2019; published 6 September 2019)

\begin{abstract}
The statistical properties of stochastic entropies in a discrete stationary one-dimensional Kardar-Parisi-Zhang system are numerically studied. As the usual time-independent solution of the associated Fokker-Planck equation is not strictly stationary, it is necessary to transform the current variables to other variables with zero spatial mean. We resorted to discrete representations in order to prove the statistical properties of entropies, and we performed a direct test of the fluctuation theorem. We discuss the connection between fluctuation theorems and the deviation from Gaussian distribution for the entropy.
\end{abstract}

DOI: 10.1103/PhysRevE.100.032111

\section{INTRODUCTION}

The Kardar-Parisi-Zhang (KPZ) equation is an emblematic model for describing the growth of surfaces [1,2] as well as a nice representation of a simple (albeit nontrivial) out-ofequilibrium extended system [3,4]. In its more generic onedimensional form, it reads

$$
\frac{\partial h(x, t)}{\partial t}=\mu \partial_{x}^{2} h(x, t)+\frac{\lambda}{2}\left[\partial_{x} h(x, t)\right]^{2}+\xi(x, t),
$$

$h(x, t)$ being the height of a given interface that grows with a combination of diffusive and nonlinear forces and driven by a Gaussian noise, uncorrelated in space and time, $\left\langle\xi(x, t) \xi\left(x^{\prime}, t^{\prime}\right)\right\rangle=2 D \delta\left(x-x^{\prime}\right) \delta\left(t-t^{\prime}\right)$. The parameters $\mu$, $\lambda$, and $D$ account for the three necessary contributions to have the generic behavior of the KPZ universality of growth. The linear version of this equation, with $\lambda=0$, corresponds to the Edwards-Wilkinson (EW) equation.

Over the past few decades, there has been exhaustive study of the interface statistical properties of KPZ models. A large variety of methods of statistical physics, ranging from scaling theories [5,6] and dynamical renormalization-group methods [7] to functional [8,9] and numerical [10-13] approaches, have been used to find the behavior of spectral densities and correlations. In recent years, interest has been focused on the possibility of finding exact results for the height probability density and the rich mathematical connection with other surprisingly related problems [14-18].

The KPZ equation is also known as being a simple but not trivial prototype of an extended out-of-equilibrium system [4]. Simple ways of getting out-of-equilibrium systems consist of introducing external fields or forces [19], colored noises [20], and other external constraints [21]. The KPZ equation is an example of a model that is intrinsically outof-equilibrium, that is, without the contribution of external mechanisms. But, despite its intrinsic interest, studies of the statistical behavior of entropies in these systems are scarce. Only the case of a deposition model whose dynamics belong to the KPZ universality class has been analyzed [22]. In such models, the three effects of diffusion, nonlinear forces, and noise-are combined, and only a reduced part of the parameter space can be analyzed. The aim of this paper is to perform a numerical analysis of stochastic entropies in the KPZ equation in its more general form, using all the stable parametric space and paying attention to both small and large systems.

\section{TIME-INDEPENDENT SOLUTIONS AND STATIONARY DISTRIBUTIONS}

The KPZ equation (1) is a space-extended version of a Langevin equation with additive noise, which means that we have a Fokker-Planck equation for $P([h(x)], t)$, the probability density of profiles $[h(x)]$ at a given time, and a functional representation of the probability density of a given process $P[h(x, s)]$. Both are important elements when analyzing a system within the framework of stochastic thermodynamics $[4,23]$. Moreover, there exists an exact time-independent solution of the Fokker-Planck equation given by $P([h(x)]) \sim$ $\exp \left(-\frac{\mu}{2 D} \int d x\left[\partial_{x} h(x)\right]^{2}\right)$, which is independent of $\lambda$, coinciding with the solution of the EW equation. But this time-independent solution is strictly a nonstationary solution for the probability density of the height, since it cannot be normalized. This is because the spatial mean value of the nonlinear term is always positive, producing a motion in the spatial mean

$$
\bar{h}(t)=\bar{h}(0)+\frac{\lambda}{2} \int_{0}^{t} d s \overline{\left[\partial_{x} h(x, t)\right]^{2}}
$$

with a sample average that grows in time as $\langle\bar{h}(t)\rangle \sim v t+$ $\sigma_{v} t^{1 / 2}$. From now on, we represent spatial averages with an overline, and sample averages with the variable between angular brackets \langle\rangle . Then a strict stationary solution for finite systems is not possible, even for the height in the moving frame $h(x, y)-v t$, whose spatial mean has a diffusive behavior. However, processes involving spatial variations, such as gradients and curvatures, could be stationary. Also, stationary solutions are possible for the variable $z(x, t)=h(x, t)-\bar{h}(t)$, 
which is driven by the modified KPZ equation

$$
\begin{aligned}
\frac{\partial z(x, t)}{\partial t}= & \mu \partial_{x}^{2} z(x, t)+\frac{\lambda}{2}\left[\partial_{x} z(x, t)\right]^{2}-\frac{\lambda}{2} \overline{\left[\partial_{x} z(x, t)\right]^{2}} \\
& +\xi(x, t) .
\end{aligned}
$$

Hence, the spatial functional

$$
P([z(x)]) \sim \exp \left(-\frac{\mu}{2 D} \int d x\left[\partial_{x} z(x)\right]^{2}\right),
$$

which is a time-independent solution of the corresponding Fokker-Planck equation that, together with the constraint $\bar{z}=0$, can now be normalized. More explicit results can be obtained in the discrete version of these equations, as we show in the next section.

\section{DISCRETE KPZ EQUATIONS}

In Eq. (2) we have assumed that the mean spatial value of the noise is zero, but in a discrete version of a finite system this mean value is nonzero and should be taken into account. Then, any spatially discrete version of (2) with $N$ spatial points in the form

$$
\dot{y}_{i}(t)=Y_{i} \mathbf{y}, t+\xi_{i}(t),
$$

with $\bar{Y}=0, Y_{i}(\mathbf{y}+\mathbf{k})=Y_{i}(\mathbf{y})$, where $\mathbf{k}$ is such that $k_{i}=k$, with $k$ an arbitrary constant, and noise correlations

$$
\left\langle\xi_{i}(t) \xi_{j}(s)\right\rangle=\frac{2 D}{a} \delta_{i, j} \delta(t-s),
$$

$a$ being the spatial step, admits a Fokker-Planck equation as

$$
\frac{\partial P(\mathbf{y}, t)}{\partial t}=-\sum_{i=1}^{N} \frac{\partial}{\partial y_{i}}\left(Y_{i} P(\mathbf{y}, t)\right)+\frac{D}{a} \sum_{i=1}^{N} \frac{\partial^{2}}{\partial y_{i}^{2}} P(\mathbf{y}, t),
$$

and also a functional form of the probability for a given path [24],

$$
P[\mathbf{y}(s)] \sim \exp \left[-\frac{a}{4 D} \int_{0}^{t} d s \sum_{i=1}^{N}\left[\dot{y}_{i}(s)-Y_{i}(\mathbf{y})\right]^{2}\right] .
$$

Note that even in the case in which there is a timeindependent solution of Eq. (5), this solution still fails to be normalized since although $\overline{\mathbf{y}}$ has a zero mean value, its variance grows diffusively in time due to the nonzero value of the mean spatial noise. In the continuous limit, where $N \rightarrow \infty$ before $t \rightarrow \infty$, the time-independent solution (3) becomes a true stationary solution. Then, to also get true stationary solutions in the discrete case, we should consider the variables $z_{i}=y_{i}-\overline{\mathbf{y}}$. A change of variables from $\left\{y_{i}\right\}$ to $\left\{\mathbf{z}^{\prime}=\left\{z_{1}, z_{2}, \ldots, z_{N-1}\right\}, \overline{\mathbf{y}}\right\}$ followed by an integration of the variable $\overline{\mathbf{y}}$ gives a Fokker-Planck equation for $P\left(\mathbf{z}^{\prime}, t\right)=$ $\int d \overline{\mathbf{y}} P\left(\mathbf{z}^{\prime}, \overline{\mathbf{y}}, t\right)$,

$$
\frac{\partial P\left(\mathbf{z}^{\prime}, t\right)}{\partial t}=-\sum_{i j}^{N-1} b_{i j} \partial_{z_{i}}\left[Y_{j} P-\frac{D}{a} \sum_{l}^{N-1} b_{j l} \partial_{z_{l}} P\right]
$$

with $b_{i j}=\delta_{i j}-\frac{1}{N}$.
Similarly, the functional form for the probability of the process $\left\{\mathbf{z}^{\prime}(s), \overline{\mathbf{y}}(s)\right\}$ becomes separable, and we have

$$
P\left[\mathbf{z}^{\prime}(s)\right] \sim \exp \left[-\frac{a}{4 D} \int_{0}^{t} d s \sum_{i=1}^{N}\left[\dot{z}_{i}(s)-Y_{i}(\mathbf{z})\right]^{2}\right],
$$

with $z_{N}(s)=-\sum_{i=1}^{N-1} z_{i}(s)$. As was mentioned previously, these are the necessary elements to analyze a system in the context of stochastic thermodynamics. Following the method of [4], we define a stochastic entropy at a time $t$ on a given realization of the process as $s_{\mathrm{sys}}(t)=-\ln \left(P\left(\mathbf{z}^{\prime}(t), t\right)\right)$. In an open system, the rate of variation of this entropy $\dot{s}(t)$ is the difference between the rate of the total entropy $\dot{s}_{T}$ and that of the exchange entropy $\dot{s}_{m}$. Total variation of the system entropy in a given realization of the process is, by definition, independent of such realization and only depends on the initial and final state, at times $t_{i}$ and $t_{f}$, respectively : $\Delta s_{\text {sys }}=$ $-\ln \frac{P\left(\mathbf{z}^{\prime}\left(t_{f}\right), t_{f}\right)}{P\left(\mathbf{z}^{\prime}\left(t_{i}\right), t_{i}\right)}$. However, the variations of the other entropies, $\Delta s_{\text {tot }}\left[\mathbf{z}^{\prime}(s)\right]$ and $\Delta s_{m}\left[\mathbf{z}^{\prime}(s)\right]$, are generally functionals of the given process. A convenient way to define these quantities is by relating them to the probabilities of forward $\left[\mathbf{z}^{\prime}(s)\right]$ and backward $\left[\overleftarrow{\mathbf{z}^{\prime}}(s)\right]=\left[\mathbf{z}^{\prime}\left(t_{f}-s\right)\right]$ trajectories. In this way, we have

$$
\begin{aligned}
\Delta s_{m}\left[\mathbf{z}^{\prime}(s)\right] & =\ln \left(\frac{P\left[\mathbf{z}^{\prime}(s)\right]}{P\left[\overleftarrow{\mathbf{z}^{\prime}}(s)\right]}\right) \\
& =\frac{a}{D} \int_{t_{i}}^{t_{f}} d s \sum_{i=1}^{N-1} \dot{z}_{i}(s)\left[Y_{i}\left(\mathbf{z}^{\prime}, s\right)-Y_{N}\left(\mathbf{z}^{\prime}, s\right)\right],
\end{aligned}
$$

and, as a consequence,

$$
\Delta s_{\mathrm{tot}}\left[\mathbf{z}^{\prime}(s)\right]=\Delta s_{m}\left[\mathbf{z}^{\prime}(s)\right]-\ln \left(\frac{P\left(\mathbf{z}^{\prime}\left(t_{f}\right), t_{f}\right)}{P\left(\mathbf{z}^{\prime}\left(t_{i}\right), t_{i}\right)}\right) .
$$

A necessary requirement of the stochastic theory is to yield the standard formulation of macroscopic quantities through the mean values of the corresponding stochastic variables. So we should have for all entropies $\Delta S_{\mathrm{sys}, m, \text { tot }}(t)=$ $\left\langle\Delta s_{\mathrm{sys}, m, \text { tot }}(t)\right\rangle$. Moreover, a stochastic theory gives additional information through the statistic properties of these variables. This information is particularly valuable for small systems and short times. From this point of view, in this paper we analyze the statistical properties of the total stochastic entropy by means of $P(q)=\left\langle\delta\left(q-\Delta s_{\text {tot }}\left[\mathbf{z}^{\prime}(s)\right]\right)\right\rangle$ as well as the exchange stochastic entropy with the analysis of $P(r)=$ $\left\langle\delta\left(r-\Delta s_{m}\left[\mathbf{z}^{\prime}(s)\right]\right)\right\rangle$ for a KPZ system in a stationary state. Note that the fluctuation theorem gives an important property to the latter distribution, stating that [3]

$$
P(q)=\exp ^{q} P(-q) .
$$

\section{NUMERICAL ANALYSIS}

Our numerical analysis is based on the spatially discrete Langevin equation (4) with periodic boundary conditions. First, it is convenient to separate the linear and nonlinear terms of the force as

$$
Y_{i}(\mathbf{y})=\mu \Gamma_{i}(\mathbf{y})+\frac{\lambda}{2}\left[\Phi_{i}(\mathbf{y})-\overline{\boldsymbol{\Phi}(\mathbf{y})}\right],
$$


taking for the linear term a symmetrical form

$$
\Gamma_{i}(\mathbf{y})=\partial_{i}^{2} \mathbf{y}
$$

with $\partial_{i} \mathbf{y}=\frac{1}{2 a}\left(y_{i+1}-y_{i-1}\right)$.

This linear contribution can be written in terms of a potential function as $\Gamma_{i}(\mathbf{y})=-\frac{\partial U(\mathbf{y})}{\partial y_{i}}$, where

$$
U(\mathbf{y})=\sum_{i=1}^{N}\left(\partial_{i} \mathbf{y}\right)^{2} .
$$

So, for the EW system $(\lambda=0)$ a solution of the timeindependent Fokker-Planck equation (5) with $\frac{\partial P(\mathbf{y}, t)}{\partial t}=0$ is

$$
P(\mathbf{y}) \sim \exp \left(-\frac{a \mu}{D} U(\mathbf{y})\right) .
$$

Note that, as mentioned before, this function cannot be normalized. A normalized solution in terms of variables $\mathbf{z}^{\prime}$ is

$$
P\left(\mathbf{z}^{\prime}\right)=C_{N} \int d z_{N} \delta(\overline{\mathbf{z}}) \exp \left(-\frac{\mu a}{D} U(\mathbf{z})\right)
$$

with $C_{N}$ given by

$$
\begin{aligned}
& N^{3 / 2}\left(\frac{\mu}{8 \pi a D}\right)^{\frac{N-1}{2}} \text { for } N \text { odd, } \\
& \left(\frac{N}{2}\right)^{3 / 2}\left(\frac{\mu}{8 \pi a D}\right)^{\frac{N-2}{2}} \text { for } N \text { even. }
\end{aligned}
$$

Next, we define two types of discretization for the nonlinear term. One is the standard form given by

$$
\Phi_{i}^{s}=\left(\partial_{i} \mathbf{y}\right)^{2},
$$

and the other is a special discretization that produces exact time-independent results for the Fokker-Planck equation (5),

$$
\Phi_{i}^{e}(\mathbf{y})=\frac{1}{3}\left[\left(\partial_{i+1} \mathbf{y}\right)^{2}+\partial_{i+1} \mathbf{y} \partial_{i-1} \mathbf{y}+\left(\partial_{i-1} \mathbf{y}\right)^{2}\right] .
$$

Note that this is not the same form given in [25] since, as seen in [26,27], it is important to maintain the same discretization criteria between the linear and nonlinear terms.

A condition for (13) to be a time-independent solution of the Fokker-Planck equation (5) is

$$
\sum_{i=1}^{N}\left(\frac{\partial \Phi_{i}}{\partial y_{i}}+\Phi_{i} \Gamma_{i}\right)=0 .
$$

Note that with standard discretization, using $\Phi_{i}^{s}$, this condition does not hold, and with exact discretization, with $\Phi_{i}^{e}$, it does. However, we have seen in our simulations that although standard discretization is not exact, it is a very good approximation for any possible value of parameters and size. We have checked numerically this surprising fact, plotting the distribution

$$
P(\theta)=\left\langle\delta\left(\theta-\frac{a \mu}{D} U(\mathbf{z})\right)\right\rangle,
$$

which, assuming that (14) is exact, gives for $P(\theta)$

$$
\begin{array}{ll}
\frac{1}{\Gamma\left(\frac{N-1}{2}\right)} \theta^{\frac{N-3}{2}} e^{-\theta} & \text { for } N \text { odd } \\
\frac{1}{\Gamma\left(\frac{N-2}{2}\right)} \theta^{\frac{N-4}{2}} e^{-\theta} & \text { for } N \text { even. }
\end{array}
$$

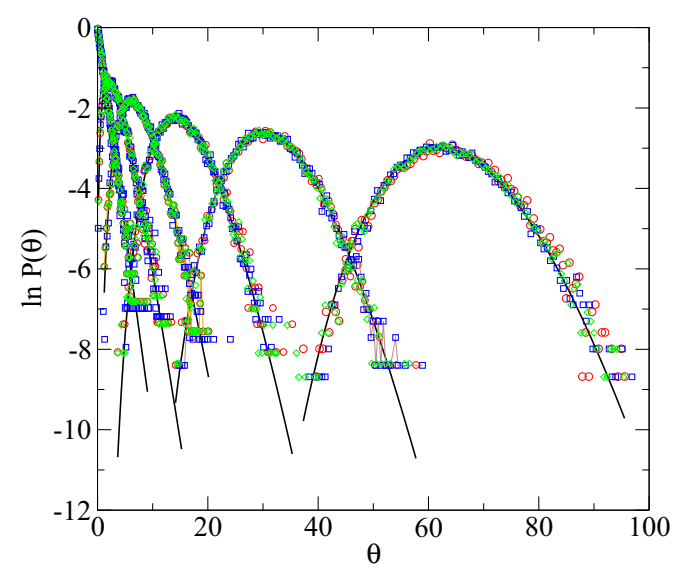

FIG. 1. Plot of $\ln P(\theta)$ vs $\theta$ for an EW model (blue squares) and a KPZ model with the exact (red circles) and standard (green diamonds) discretization compared with their analytical exact expression (dashed black lines) (17). The size of the systems goes (from left to right) from $N=4$ to 128 in powers of 2 . Model parameters are $\mu=1, D=0.01$, and $\lambda=1$ (E1 and S1) for the KPZ model.

One would expect good agreement between numerical simulations and this expression in the second discretization case, where (14) is exact, and also close to the continuous limit in the standard discretization case, i.e., systems with large $N$. However, as seen in Fig. 1, there is excellent agreement in all cases, even with the smallest possible system with $N=4$.

Finally, substituting (12) into (9) and (10) and integrating the linear term, we obtain for the stochastic entropies

$$
\begin{aligned}
\Delta s_{m}\left[\mathbf{z}^{\prime}(s)\right]= & \frac{a \lambda}{2 D} \int_{t_{i}}^{t_{f}} d s \sum_{i=1}^{N-1} \dot{z}_{i}(s)\left[\Phi_{i}\left(\mathbf{z}^{\prime}(s)\right)-\Phi_{N}\left(\mathbf{z}^{\prime}(s)\right)\right] \\
& +\frac{a \mu}{D}\left[U\left(\mathbf{z}\left(t_{i}\right)\right)-U\left(\mathbf{z}\left(t_{f}\right)\right)\right] \\
\Delta s_{\mathrm{tot}}\left[\mathbf{z}^{\prime}(s)\right]= & \frac{a \lambda}{2 D} \int_{t_{i}}^{t_{f}} d s \sum_{i=1}^{N-1} \dot{z}_{i}(s)\left[\Phi_{i}\left(\mathbf{z}^{\prime}(s)\right)-\Phi_{N}\left(\mathbf{z}^{\prime}(s)\right)\right]
\end{aligned}
$$

\section{A. Numerical setup}

We simulate the Langevin equation (4) with periodic boundary conditions $y_{0}=y_{N}, y_{N+1}=y_{1}$, and prepoint discretization in time,

$$
y_{i}\left(t_{j+1}\right)=y_{i}\left(t_{j}\right)+v Y_{i}\left(\mathbf{y}\left(t_{j}\right)\right)+\sqrt{\frac{2 D v}{a}} \xi_{i, j},
$$

taking as time and space steps $v=0.01$ and $a=1$, respectively, and $\xi_{i, j}$ being independent normalized Gaussian noises. The initial state to compute the stochastic entropies (19) and (18) is reached after evolving (20) during a long enough time to ensure saturation of the interface width. Distributions $P(q)$ and $P(r)$ are obtained as a normalized histogram (using 10000 samples) of the stochastic entropies taking (14) as the stationary distribution. All simulations are performed with both the standard (S) and exact (E) discretization. To have a wide sampling of the parameter space, we take two extreme values of parameters $\lambda$ and $D$, labeled as (i) $\lambda=1, D=$ 

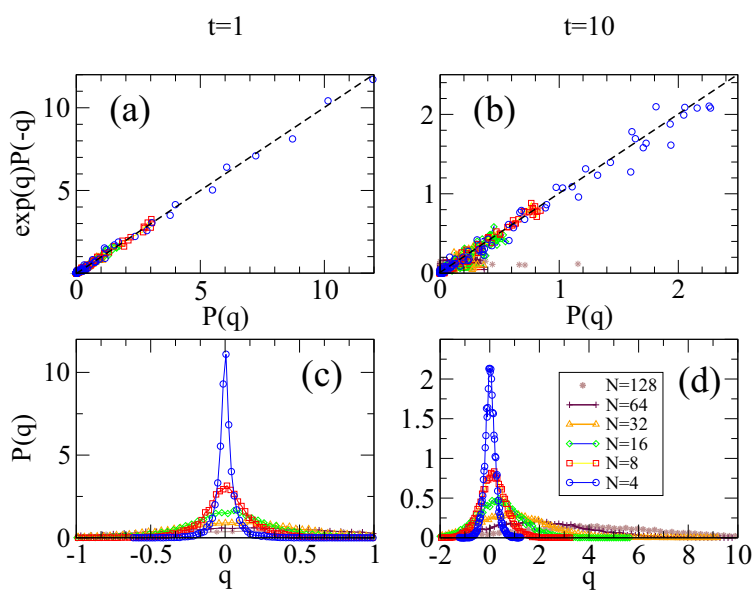

FIG. 2. Upper row: plot of $\exp (q) P(-q)$ vs $P(q)$ in $t=1$ (a) and $t=10$ (b) for a direct checking of the fluctuation theorem. Lower row: probability density $P(q)$ in $t=1$ (c) and $t=10$ (d). The model is $\mathrm{KPZ}$ with the standard discretization and parameters $\mu=\lambda=1$, $D=0.01(\mathrm{~S} 1)$.

0.01 and (ii) $\lambda=0.1, D=1$. The parameter $\mu$ varies above the minimum value to have stable growth. We take $\mu_{\min }=$ $\{0.8,1,1.2,1.4,1.6,1.8\}$.

\section{B. Small systems and short times: A direct check of the fluctuation theorem}

A direct check of the fluctuation theorem, for instance plotting $e^{q} P(-q)$ versus $P(q)$ as in Fig. 2, is only possible for small systems and short times, where the negative part of the distribution is still a central part, and statistical errors are not significant. As we can see in such a figure, the theorem is perfectly observed for $t=1$ in all system sizes, from $2^{3}$ to $2^{7}$, whereas for $t=10$ it is only observed in sizes below $2^{4}$.

\section{Mean values of entropies}

From (19) and (18) one immediately sees that the mean values of both entropies,

$$
\begin{aligned}
q_{m}(t) & =\int q P(q) d q=\left\langle\Delta s_{\mathrm{tot}}\left[\mathbf{z}^{\prime}(s)\right]\right\rangle, \\
r_{m}(t) & =\int r P(r) d r=\left\langle\Delta s_{m}\left[\mathbf{z}^{\prime}(s)\right]\right\rangle,
\end{aligned}
$$

are identical, $q_{m}(t)=r_{m}(t)$. A plot of these values versus time for distinct system sizes is shown in Fig. 3. A perfect linear dependence for any time and any size is observed. Taking into account this fact and the extensive character of entropies, we have

$$
q_{m}(t)=r_{m}(t)=\rho_{\dot{s}}(N) a N t,
$$

where $\rho_{\dot{s}}(N)$ is the entropy production density, that is, the entropy production per unit of size. Numerical simulations indicate that this quantity varies as

$$
\rho_{\dot{s}}=C(N) \frac{D \lambda^{2}}{a^{2} \mu^{2}},
$$
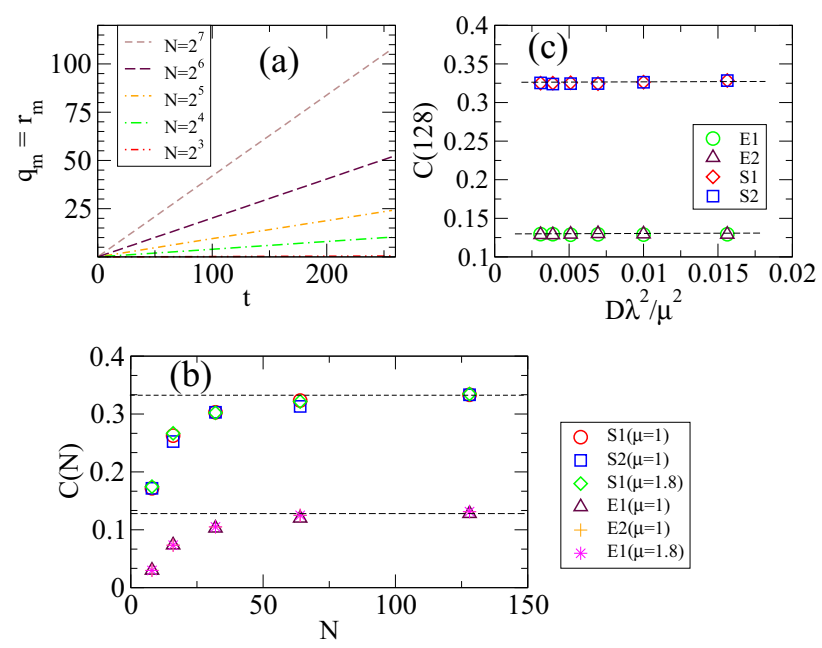

FIG. 3. (a) Mean stochastic entropies $q_{m}=r_{m}$ as a function of time for a KPZ model ( $\mathrm{S} 1$ with $\mu=1$ ) with sizes in increasing slopes from $N=2^{3}$ to $2^{7}$ in successive powers of 2. (b) $C(N)$ as a function of the system size for a set of parameters. (c) Saturation values of the dimensionless parameter $C$ for a wider set of system parameters with $\mu=\{0.8,1,1.2,1.4,1.6,1.8\}$.

$C(N)$ being dependent of the discretization type and growing exponentially with size until saturation to a value $C(\infty)$, where $C(\infty) \sim 0.325$ or $C(\infty) \sim 0.129$ for the standard or exact discretization, respectively (Fig. 3). Note that this value is dimensionless since the entropy production density has dimensions of $[x]^{-1}[t]^{-1}$, which is the same dimension of $\frac{D \lambda^{2}}{\mu^{2} a^{2}}$. But the entropy production density is not a universal quantity in the sense of Krug [28]. On the one hand, although a dimensionless constant exists, here it depends on the discretization. On the other hand, the entropy production density depends explicitly on the spatial step $a$. Note that this would be expected since, in general, the entropy in continuous physical systems requires a cutoff to be properly defined [4]. However, although Eq. (22) cannot be universal, it indicates some kind of regularity.

\section{Deviation from Gaussian distributions and the fluctuation theorem}

The fluctuation theorem (11) is the cause of a strong symmetry constraint on the distribution of stochastic entropy $P(q)$ that creates a very interesting relationship between the mean and the variance [29]. If such a distribution were Gaussian, of the form $P(q) \sim \exp \left(-\frac{\left(q-q_{m}\right)^{2}}{2 \sigma_{q}^{2}}\right)$, this symmetry would immediately imply a value 2 for the variance to mean ratio, $\sigma_{q}^{2}=2 q_{m}$. Numerical values of this ratio deviate slightly from 2 , indicating that $P(q)$ also deviates slightly from the Gaussian distribution. As shown in the right panel of Fig. 4, for long enough times and large sizes we have

$$
\sigma_{q}^{2}(t)=2(1+\epsilon) q_{m}(t),
$$

$\epsilon$ being a parameter that gives a measure of the deviation of the distribution function from a Gaussian. In the left panel of this figure (upper part), we show that this deviation is proportional to the entropy production density. To confirm such a deviation 


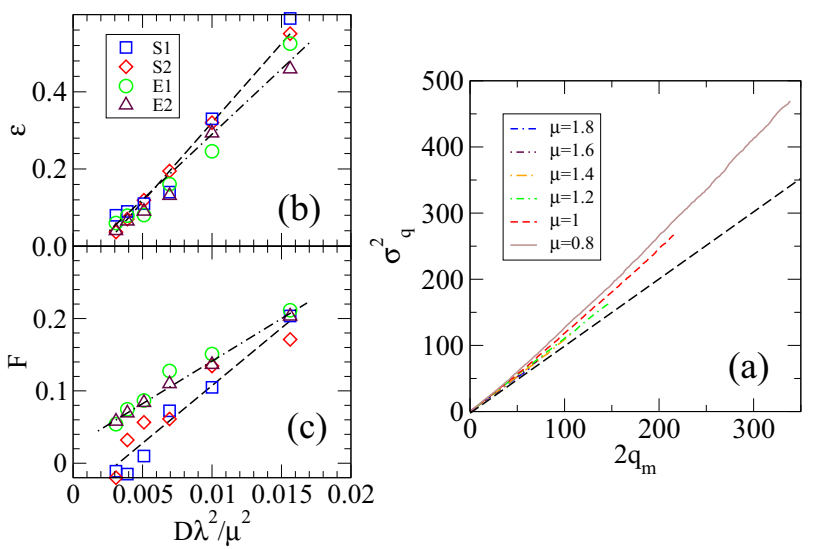

FIG. 4. (a) Plot of total entropy variance vs double mean in time of a KPZ (S1) model to show the Gaussian deviation for distinct values of the diffusive parameter $\mu$. Assuming the fluctuation theorem, the Gaussian behavior is represented by the black dashed line. The asymptotic Gaussian deviation (b) and the Fisher coefficient (c) are proportional to $\sim D \lambda^{2} / \mu^{2}$.

using a more standard quantifier, we compute the skewness of the distribution through the Fisher coefficient $F=\frac{\left\langle\left(q-q_{m}\right)^{3}\right\rangle}{\left\langle\left(q-q_{m}\right)^{2}\right\rangle^{3 / 2}}$, obtaining the same result: it seems to be proportional to the entropy production density (Fig. 4). Once again this proportionality depends on the type of discretization.

It would also be interesting to investigate more deeply this class of distributions. Note that assuming an exponential form, $P(q) \sim \exp (-f(q))$, and separating $f(q)$ into odd and even functions, $f_{\text {odd }}+f_{\text {even }}$, the fluctuation theorem imposes the
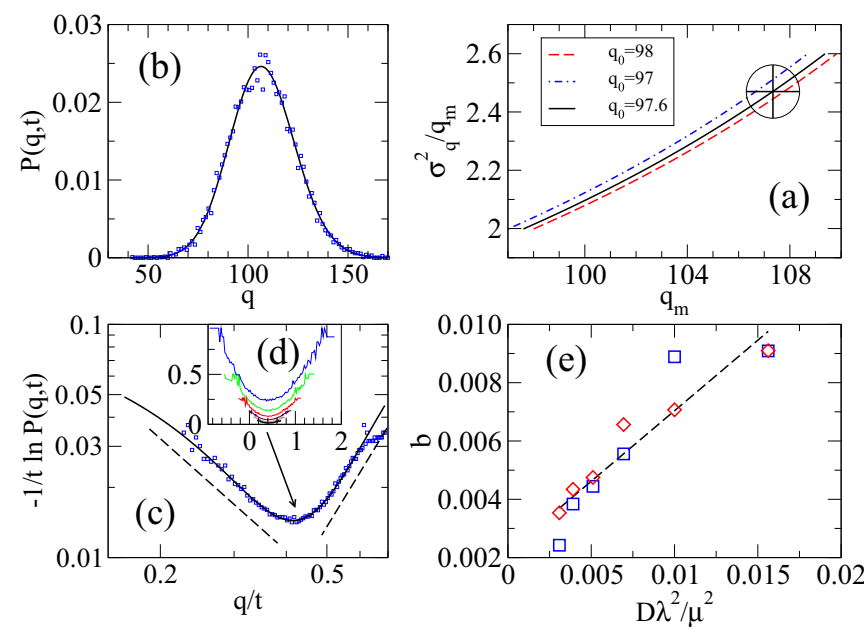

FIG. 5. (a) Choosing parameters of the quartic model for a KPZ (S1) system with $\mu=1, N=128, t=257$. (b) Probability densities $P(q)$ of the simulated KPZ model (blue squares) and its fitted quartic model (continuous black line) with $q_{0}=97.6, b=0.0088$. (c) Large deviation function in a log-log plot. The dashed lines correspond to tails in the function of $x^{-1.70}$ (left) and $x^{2.70}$ (right). (d) In ordinary coordinates, the large deviation function appears in the limit of large $t$. The functions go downward from $t=2^{3}$ to $2^{8}$ in powers of 2 . (e) The quartic parameter $b$ is proportional to the entropy production density in an S1 (red diamond) and S2 (blue square) parametrization scheme with $\mu=\{0.8,1,1.2,1.4,1.6,1.8\}$.

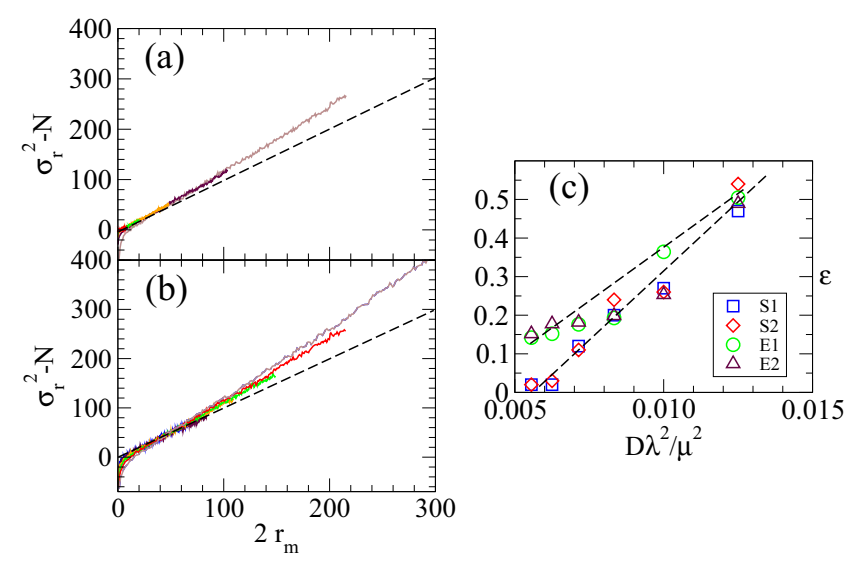

FIG. 6. (a) Plot of $\sigma_{r}^{2}-N$ vs $2 r_{m}$ for sizes between $2^{3}$ and $2^{8}$ in powers of 2 , and (b) $\mu=\{0.8,1,1.2,1.4,1.6,1.8\}$. (c) Asymptotic slopes as a function of $D \lambda^{2} / \mu^{2}$.

form of the odd function as $f_{\text {odd }}=\frac{1}{2} q$. A simple model to describe the slight deviation from the Gaussian distribution consists in considering a quartic correction,

$$
p(q) \sim \exp \left[-\frac{\left(q-q_{0}\right)^{2}}{4 q_{0}}+b\left(\frac{q^{4}}{q_{0}^{3}}\right)\right],
$$

and adjusts the parameters $q_{0}$ and $b$ to reproduce the numerical distribution in a large enough interval (albeit bounded) around the mean value. In Fig. 5 we show the implementation of this scheme in a given case. The parameters are chosen to exactly reproduce the mean $q_{m}$ and the variance to mean ratio $\sigma_{q}^{2} / q_{m}$. In these conditions, the probability density is very well reproduced (see the right panel of Fig. 5) with a less than $2 \%$ error of the Fisher coefficient $F=0.13$. To see the asymmetry of the distribution in a graphical form, in Fig. 5 we also show (lower panels) the shape of the large deviation function in $\log -\log$ coordinates [30]. Note that to quantify the deviation from the Gaussian distribution, we have analyzed three parameters: $\epsilon, F$, and $b$. An interesting fact is that they are all proportional to the entropy production density, with a constant that changes with the discretization.

\section{E. Scaling of the stochastic exchange entropy}

The distribution of the stochastic exchange entropy $P(r)$ is always Gaussian. Values of the skewness $F$ in all cases are below 0.01 . However, there exists some connection between the values of variances of both entropies. As shown in Fig. 6, the following scaling law can be extracted from the numerical data:

$$
\sigma_{r}^{2}=2(1+\epsilon) r_{m}+A N,
$$

where $A \sim 1$ in all simulated cases. As indicated before, the values of $\epsilon$ are proportional to the production entropy density.

\section{CONCLUSIONS}

In this work, we studied a discrete one-dimensional KPZ system in order to obtain information on the statistical properties of stochastic entropies. To achieve this, we exploited some numerical methods. As the time-independent solution 
of the associated KPZ equation is not strictly stationary (i.e., it is non-normalizable), we had to resort to a transformation of variables to an "inertial system," which enabled us to obtain a related equation that has a true stationary probability distribution function (PDF). By analyzing discrete versions of the involved equations, we were able to study the indicated entropy statistical properties.

As a check of the discretization methods, we studied (see Fig. 1) the PDF $P(\theta)$, i.e., the stationary distribution of the potential $\frac{\mu}{D} U[z]$, for diverse system sizes. We found excellent agreement between theory, indicated by Eq. (17), and our numerical analysis. It is worth remarking that even for a size of $N=4$, there is still excellent agreement.

In Fig. 2, we depict a direct test of the fluctuation theorem, Eq. (11). Although in principle such a relation is only observable for small systems, it is apparent that for short times it is also observable for relatively large systems. For longer times, such an observation is reduced to smaller systems.

In Fig. 3, we show numerically the parameter dependence of the entropy production density, which only depends on discretization through a dimensionless constant.

As is well known, the fluctuation theorem, Eq. (11), induces a strong symmetry constraint on the distribution of the stochastic entropy $P(q)$ that, when Gaussian, produces a deviation to a mean ratio equal to 2 . Numerical results shown in Fig. 4 clearly indicate the existence of a departure from a Gaussian behavior for such a distribution. In Fig. 5 it is shown that such a departure is adequately taken into account by a quartic correction, which is compatible with the fluctuation theorem and valid in the numerically observed interval around the mean value. To quantify this departure, together with the quartic parameter $b$ we also show the value of the skewness of the distribution. All departure quantifiers are proportional to the entropy production density. More graphical information is also included in Fig. 5 by showing the asymmetry of the large deviation function. Finally, for the sake of comparison, the scaling behavior of the variance to mean ratio for the exchange entropy distribution is shown in Fig. 6.

\section{ACKNOWLEDGMENTS}

M.A.R. acknowledges support from the Ministerio de Economia y Competitividad (MINECO) under Projects No. Fis2016-74957-P and No. CGL2015-66583R. H.S.W. thanks the IFISC for the kind hospitality extended to him.
[1] M. Kardar, G. Parisi, and Y. C. Zhang, Phys. Rev. Lett. 56, 889 (1986).

[2] T. Halpin-Healy and Y.-C. Zhang, Phys. Rep. 254, 215 (1995).

[3] U. Seifert, Rep. Prog. Phys. 75, 126001 (2012).

[4] U. Seifert, Phys. Rev. Lett 95, 040602 (2005).

[5] J. Krug and H. Spohn, in Kinetic Roughening of Growing Surfaces, in Solids Far from Equilibrium, edited by C. Godreche (Cambridge University Press, Cambridge, 1992).

[6] J. Krug, Adv. Phys. 46, 139 (1997).

[7] A. L. Barabasi and H. E. Stanley, Fractal Concepts in Surface Growth (Cambridge University Press, Cambridge, UK, 1995).

[8] H. C. Fogedby, Phys. Rev. Lett. 80, 1126 (1998).

[9] H. S. Wio, M. A. Rodriguez, R. Gallego, R. R. Deza, and J. A. Revelli, Front. Phys. 4, 52 (2017).

[10] T. Halpin-Healy, Phys. Rev. Lett. 109, 170602 (2012).

[11] T. Halpin-Healy, Phys. Rev. E 88, 042118 (2013).

[12] R. Gallego, M. Castro, and J. M. Lopez, Phys. Rev. E 76, 051121 (2007).

[13] L. Giada, A. Giacometti, and M. Rossi, Phys. Rev. E 65, 036134 (2002).

[14] T. Halpin-Healy and K. A. Takeuchi, J. Stat. Phys. 160, 794 (2015).

[15] M. Prahofer and H. Spohn, Phys. Rev. Lett. 84, 4882 (2000).

[16] P. L. Ferrari and H. Spohn, Commun. Math. Phys. 265, 1 (2006).
[17] M. Hairer, Ann. Math. 178, 559 (2013).

[18] P. Calabrese and P. Le Doussal, Phys. Rev. Lett. 106, 250603 (2011).

[19] K. Turitsyn, M. Chertkov, V. Y. Chernyak, and A. Puliafito, Phys. Rev. Lett. 98, 180603 (2007).

[20] M. K. Sen, A. Baura, and B. C. Bag, Eur. Phys. J. B 83, 381 (2011).

[21] T. Taniguchi and E. G. D. Cohen, J. Stat. Phys. 126, 1 (2007).

[22] A. C. Barato, R. Chetrite, H. Hinrichsen, and D. Mukamel, J. Stat. Mech. (2010) P10008.

[23] V. Y. Chernyak, M. Chertkov, and C. Jarzynski, J. Stat. Mech. (2006) P08001.

[24] To avoid unnecessary complications, we always assume a prepoint time discretization of the Langevin equations, which gives a value 1 in the Jacobian of the transformation $\xi \rightarrow \mathbf{y}$.

[25] C. H. Lam and F. G. Shin, Phys. Rev. E 58, 5592 (1998).

[26] H. S. Wio et al., EPL 89, 40008 (2010).

[27] H. S. Wio, J. A. Revelli, R. R. Deza, C. Escudero, and M. S. de La Lama, Phys. Rev. E 81, 066706 (2010).

[28] J. Krug, P. Meakin, and T. Halpin-Healy, Phys. Rev. A 45, 638 (1992).

[29] N. Merhav and Y. Kafri, J. Stat. Mech. (2010) P12022.

[30] H. Touchette, Phys. Rep. 478, 1 (2009). 